\title{
DC Microgrid Operational Method for Enhanced Service Reliability Using DC Bus Signaling
}

\author{
Pyeong-Ik Hwang*, Gilsoo Jang*, Gi-Chan Pyo**, Byung-Moon Han***, \\ Seung-Il Moon ${ }^{\S}$ and Seon-Ju Ahn ${ }^{\dagger}$
}

\begin{abstract}
This paper proposes a DC microgrid operational strategy and control method for improved service reliability. The objective is to supply power to as many non-critical loads as possible, while providing an uninterrupted power supply to critical loads. The DC bus signaling method, in which DC voltage is an information carrier, is employed to implement the operational strategy in a decentralized manner. During grid-connected operation, a grid-tied converter balances the power of the microgrid by controlling the DC voltage. All loads are connected to the microgrid, and operate normally. During islanded operation, distributed generators (DGs), a backup generator, or an energy storage system balances the power. However, some non-critical loads may be disconnected from the microgrid to ensure the uninterrupted power supply to critical loads. For enhanced service reliability, disconnected loads can be automatically reconnected if certain conditions are satisfied. Control rules are proposed for all devices, and detailed microgrid operational modes and transition conditions are then discussed. Additionally, methods to determine control parameter settings are proposed. PSCAD/EMTDC simulation results demonstrate the performance and effectiveness of the proposed operational strategy and control method.
\end{abstract}

Keywords: Autonomous operation, DC bus signaling, DC microgrid, Decentralized operation

\section{Introduction}

In recent years, distributed generator (DG) technology has emerged as a solution to energy and environmental problems, such as global warming, depletion of fossil fuel resources, and growth of energy demand [1,2]. Although DGs offer many advantages to a power system, they can cause problems such as voltage rise and protection issues as DG penetration increases [3]. The concept of a microgrid has been proposed to solve these problems [4]. A microgrid is defined as an independent distribution network comprising various DGs, energy storage systems (ESSs), and controllable loads [5].

There are two different microgrid concepts: the AC microgrid and the DC microgrid. Most systems adopt the AC microgrid concept, because it can utilize existing AC grid technologies, protection schemes, and standards [6, 7]. However, the DC microgrid concept has been introduced as the more suitable interconnection concept for DC loads and DC output DGs, such as photovoltaic systems, fuel cells,

$\dagger$ Corresponding Author: Dept. of Electrical Engineering, Chonnam National University, Korea. (sjahn@jnu.ac.kr)

* School of Electrical Engineering, Korea University, Korea. (hpi@ powerlab.snu.ac.kr, gjang@korea.ac.kr)

** Machinery \& Electric System Team, Samsung Heavy Industries, Korea. (pyogichan@gmail.com)

*** Dept. of Electrical Engineering, Myongji University, Korea. (erichan@mju.ac.kr)

$\S$ School of Electrical Engineering and Computer Science, Seoul National University, Korea. (moonsi@plaza.snu.ac.kr)

Received: August 7, 2013; Accepted: September 4, 2014 and batteries. The DC/AC and AC/DC conversion systems required in an $\mathrm{AC}$ microgrid can be eliminated in a $\mathrm{DC}$ microgrid. Therefore, the efficiency of energy conversion is increased, and the implementation cost and system size are reduced. Moreover, synchronization and reactive power problems, which are inherent drawbacks of an $\mathrm{AC}$ grid, do not occur in a DC microgrid $[8,9]$.

Operational methods for DC microgrids are divided into centralized and decentralized methods. In a centralized operational method, energy sources and loads are controlled by a central control system, and thus a fast communication system is necessary due to the low inertia (determined by the capacity of the capacitors in the DC microgrid). With this type of operational method, it is easy to implement various operational strategies, and to achieve optimal operation of a DC microgrid. However, the implementation cost is increased, and the reliability of the system is degraded by the communication system [10]. Various operational strategies and control methods have been proposed for centralized operational methods [9, 11-13]. In [13], a voltage clamp control was proposed to overcome the degradation of reliability caused by the communication system. In a decentralized operational method (also known as an autonomous operational method), the energy sources and loads change their states and operate according to their terminal quantities, and thus a communication system is not indispensable. Therefore, reliability is improved and the implementation cost can be reduced, in comparison to a centralized operational method 
[10]. Moreover, a decentralized method can be used as a backup control method for a centralized method, when the communication and / or central control system are unavailable. A power-sharing method among energy sources, based on a droop control, was proposed and analyzed in [14]. An operational method for a DC microgrid with variable, non-dispatchable generators and an ESS was proposed in [15], and a frequency-response study and experimental results were reported in [16]. A load-control method based on the voltage level of the DC bus was proposed in [17] to ensure a reliable power supply for high-priority loads. DC bus signaling (DBS) was proposed in [10] as a power-sharing method among energy sources. In DBS, the DC voltage level is utilized as an information carrier. A DBS-based operational method for integrating modular photovoltaic (PV) generation systems with battery energy storage, and a PV voltage controller was presented in $[18,19]$. In these studies, the normal operating state of the PV was maximum power point tracking (MPPT), but the PV operated in a voltage control mode if its power output was greater than the loads, and thus was forced to decrease its output to balance the active power of the DC microgrid.

We focus on a DC microgrid that ensures an uninterrupted power supply to sensitive and critical loads. Even though high reliability is required for such a DC microgrid, few researchers have proposed an operational method based on the decentralized concept [10, 17]. Moreover, the reconnection of the disconnection load, which is essential to improve service reliability, has rarely been considered. In this paper, a DBS-based operational strategy, state control method of the backup generator, and disconnection rule of the non-critical loads are proposed for an uninterrupted power supply to the critical loads. The reconnection rule of the non-critical load is also proposed to improve service reliability of non-critical loads. The remainder of the paper is divided into six sections. In Section 2, the DBS concept is described, and its characteristics are analyzed using a simplified system. Section 3 presents the proposed DC microgrid operational strategy for enhanced service reliability, as well as control rules for all devices to implement this operational strategy. In Section 4, detailed DC microgrid operational modes and transition conditions are discussed. The determination methods for the control parameters are presented in Section 5. In Section 6, the effectiveness of the proposed operational strategy and state control method are validated by PSCAD/EMTDC simulations. Finally, Section 7 contains concluding remarks.

\section{DC Bus Signaling}

DBS utilizes DC voltage as an information carrier for power sharing among dispatchable energy sources in a DC power system. The dispatchable energy sources

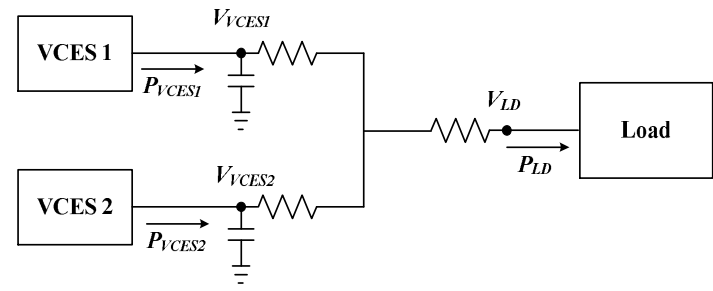

Fig. 1. Simplified DC microgrid

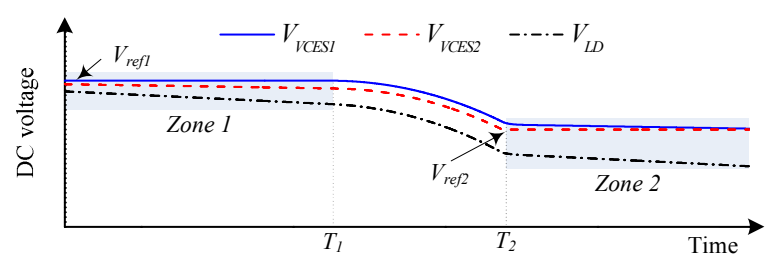

(a) DC voltages

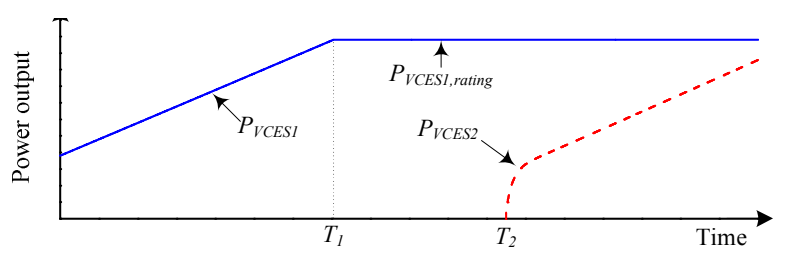

(b) Output power of the VCESs

Fig. 2. Operational characteristics of the simplified DC microgrid when the load increases gradually

independently control the DC voltage with different reference voltages. According to the kind of voltage controller used for the dispatchable energy sources, DBS methods are divided into two types: current-versus-voltage (V-I) droop control [10] and proportional-integral (PI) control [18]. In this study, PI control is utilized to implement the proposed operational strategy.

A simplified DC microgrid with two voltage-controlling energy sources (VCESs) and a load, shown in Fig. 1, is used to analyze the operational characteristics of the PIcontrol-based DBS method. Fig. 2 illustrates how the DC voltage and the power output of each VCES change when the load increases gradually. It is assumed that the reference voltage of VCES $1\left(V_{\text {refl }}\right)$ is higher than that of VCES $2\left(V_{\text {ref } 2}\right)$, and that power is initially supplied to the load by VCES 1 .

As Fig. 2(a) indicates, because of line resistance, DC voltages are not constant, but are maintained within certain ranges. The operational ranges of DC voltages are classified into two zones: Zone 1 and Zone 2. Prior to time $T_{1}$, power is supplied to the increasing load by VCES 1, and the DC voltages are maintained near $V_{\text {refl }}$ (Zone 1 ). Since the power output of VCES 1 reaches its power rating ( $\left.P_{V C E S 1, \text { rating }}\right)$ at $T_{1}$, VCES 1 cannot increase its power output any further to control the DC voltage. Between times $T_{1}$ and $T_{2}$, the increasing load is supplied with the energy stored in the capacitors, and thus the DC voltage 
decreases. At time $T_{2}$, the terminal DC voltage of VCES 2 reaches $V_{\text {ref } 2}$. After time $T_{2}$, VCES 2 starts supplying power to control the DC voltage, as shown in Fig. 2(b), and the DC voltages are maintained in Zone 2.

From this analysis, we can derive three important operational characteristics of the PI-controller-based DBS method. Firstly, the generation priority of the VCESs can be arranged by setting the reference voltages differently. The VCES with the highest reference voltage initially supplies power to the loads. Secondly, there are specific operational zones for the DC voltages, depending on the reference voltages of the VCESs. Finally, the operational reserves of the VCESs can be estimated in terms of the DC voltage; i.e., the minimum values of the upward and downward power reserves can be determined for each operational zone. The upward / downward power reserve of the microgrid is defined as the sum of the increasable/ reducible power margins of the VCESs. For example, if the DC voltage is maintained in Zone 2, the power output of VCES 1 is its power rating, and that of VCES 2 is unknown. Therefore, the minimum upward power reserve is zero, and the minimum downward power reserve is the power rating of VCES 1 . The last characteristic is very important in the implementation of the proposed strategy since the loads and DGs can estimate the states of the VCESs only in terms of the measured DC voltage.

\section{Operational Strategy and Control Rules for Devices}

Fig. 3 shows the configuration of a typical DC microgrid including critical loads. Power is supplied to the loads from the AC grid via the grid-tied converter (GTC), ESS, backup generator (BG), and DGs. In this study, the DGs are assumed to be partially dispatchable generators that can control the DC voltage by only reducing the power outputs $[18,19]$. The loads are divided into critical and non-critical loads according to their importance. The operational objective of the DC microgrid is to supply uninterrupted power to the critical loads.

A DC microgrid is normally connected to an AC grid (i.e., grid-connected operation). During normal grid-

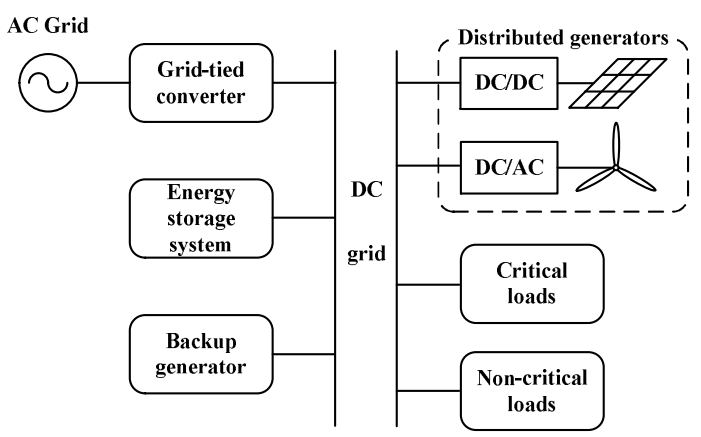

Fig. 3. Configuration of a DC microgrid connected operation, the BG is switched off, due to its high generation cost. If an outage occurs in the $\mathrm{AC}$ grid, the $\mathrm{DC}$ microgrid is disconnected from the $\mathrm{AC}$ grid and initiates islanded operation. During islanded operation, the DGs, BG, and/or ESS supply power to the loads according to the load demand.

For uninterrupted power supply to all loads, the power ratings of the GTC, BG, and ESS should all be greater than the maximum power consumption of the loads. However, this is not appropriate from an economic perspective. In this study, we assume that the power rating of the GTC $\left(P_{G T C, \text { rating }}\right)$ is greater than both the maximum power consumption of the loads and the total power rating of DGs:

$$
P_{G T C, \text { rating }}>\max \left\{\sum_{i} P_{L D, \max , i}, \sum_{j} P_{D G, \text { rating }, j}\right\}
$$

where $P_{L D, \text { max }, i}$ and $P_{D G \text {,rating, } j}$ are the maximum power consumption of load $i$ and the rated power output of DG $j$, respectively. On the other hand, the power ratings of the BG $\left(P_{B G \text {,rating }}\right)$ and the ESS $\left(P_{E S S, \text { rating }}\right)$ are only greater than the maximum power consumption of the critical loads to ensure an uninterrupted power supply to the critical loads:

$$
P_{B G, \text { rating }}, P_{E S S, \text { rating }}>\sum_{i} P_{C L D, \max , i}
$$

where $P_{C L D, \max , i}$ is the maximum power consumption of critical load $i$. Since it takes time for the BG to detect islanded operation and start generation, the ESS should be capable of supplying its rated power during this period:

$$
E_{\text {max }}-E_{\text {min }}>\left(T_{B G, \text { on }}+T_{B G, \text { start }}\right) P_{E S S, \text { rating }}
$$

where $E_{\max }$ and $E_{\min }$ are operational maximum and minimum limits of the energy stored in the ESS, respectively. $T_{B G \text { on }}$ is the time that takes to detect islanded operation, and $T_{B G \text { start }}$ is the minimum startup time of the BG. It is assumed that the power and energy capacities of the ESS are slightly greater than the minimum conditions given by (2) and (3) to reduce cost.

With these energy source ratings, power can be supplied to all loads during grid-connected operation. However, during islanded operation, power can only be supplied to the critical loads and some of the non-critical loads. The objective of the proposed operational strategy is to provide uninterrupted power to the critical loads, while shedding the minimum number of non-critical loads during islanded operation.

\subsection{Power sharing with DBS}

As explained in Section 2, the priority among VCESs in supplying power is determined by the reference voltages. For the economic operation, the DGs have the highest priority. In order to maintain power supplies to the loads 


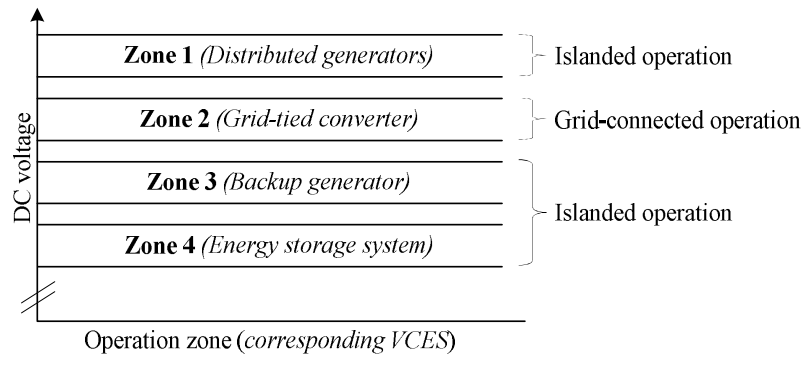

Fig. 4. Operational zones and corresponding VCESs

for as long as possible during islanded operation, the energy stored in the ESS should be kept as close as possible to its maximum during grid-connected operation. Therefore, in normal grid-connected operation, the GTC has the second priority and balances the power mismatch between the loads and DGs, while the BG is switched off and the ESS is idle and at full capacity. Since the stored energy in the ESS is limited, it is reasonable for the BG to preferentially supply power to the loads in the islanded operation. Therefore, the third priority is given to the BG, and the ESS provides power to the DC microgrid only if the other energy sources are unable to supply power to the loads.

Fig. 4 summarizes the power-sharing principle, showing the operational zones and corresponding VCESs:

$$
V_{D G s}^{*}>V_{G T C}^{*}>V_{B G}^{*}>V_{E S S}^{*}
$$

where $V_{D G s}^{*}, V_{G T C}^{*}, V_{B G}^{*}$, and $V_{E S S}^{*}$ denote the reference voltages of the DGs, GTC, BG, and ESS, respectively. The DC voltage is normally maintained in Zone 2 during gridconnected operation, and in Zone 1, Zone 3, or Zone 4 during islanded operation. Even though all DGs have same voltage reference, the loads are automatically shared among the DGs by the line resistances in Zone 1 [14].

With the proposed power-sharing principle, the ESS can automatically recover stored energy if other energy sources are able to supply power to the loads. If the remaining power of the other VCESs exceeds the maximum charging power of the ESS, the DC voltage will be maintained in Zone 2 (grid-connected operation) and Zone 1 or Zone 3 (islanded operation). Otherwise, all remaining power will be used to charge the ESS, and the DC voltage will be maintained in Zone 4.

\subsection{State control of the backup generator}

If the DC microgrid is islanded due to an outage in the $\mathrm{AC}$ grid, and thus the $\mathrm{DC}$ voltage is maintained in Zone 1 by the DGs or Zone 4 by the ESS, the BG should start generating to improve service reliability. Therefore, if the DC voltage is maintained in Zone 1 or Zone 4 for a specified time period $\left(T_{B G, o n}\right)$, the $\mathrm{BG}$ should start generating. However, when there is a short fault in the $\mathrm{AC}$ grid, the DC microgrid remains connected to the AC grid, and the DC voltage may be controlled in Zone 1 or Zone 4 . In this case, there is no need to start the BG. To prevent such unnecessary BG startups, $T_{B G, o n}$ is assigned a value slightly greater than the minimum fault duration that causes a transition to islanded operation $\left(T_{\text {faul }, \text { min }}\right)$.

\section{- BG on rule:}

$$
V_{d c} \in\{\text { Zone } 1 \bigcup \text { Zone } 4\} \text { for } T_{B G, o n}>T_{\text {fault,min }}
$$

If the fault is eliminated and the DC microgrid is reconnected to the $\mathrm{AC}$ grid, the $\mathrm{BG}$ will be switched off. However, the BG switch-off should be postponed until the ESS is replenished to specific level. Otherwise, some critical loads may be interrupted in the unlikely event that the DC microgrid is islanded once again. However, since the BG cannot estimate the exact state of the ESS, we propose the most conservative rule for switching off the BG. The BG can be switched off if the DC voltage remains in Zone 2 for the specified time period $\left(T_{B G, \text { off }}\right)$ required to bring the ESS to full charge $\left(E_{\max }\right)$ from its minimum energy $\left(E_{\min }\right)$.

\section{- BG off rule:}

$$
V_{d c} \in \text { Zone } 2 \text { for } T_{B G, \text { off }}>\frac{E_{\max }-E_{\text {min }}}{P_{E S S, \text { rating }}}
$$

\subsection{State control of non-critical loads}

During islanded operation, the DC voltage decreases when the energy sources have insufficient power to control the DC voltage [15]. In such conditions, non-critical loads should be disconnected from the DC microgrid to prevent total system collapse. The DC voltage can be also decreased during transient, even though the energy sources have enough power to supply the loads. In order to avoid the unnecessary disconnection in such transient low voltage conditions, a non-critical load is disconnected if the DC voltage is lower than a specific value $\left(V_{N L D, \text { discon }}\right)$ for a specific time $\left(T_{N L D, \text { discon }}\right)$.

\section{- Non-critical load disconnection rule:}

$$
V_{d c}<V_{N L D, \text { discon }} \text { for } T_{N L D, \text { discon }}
$$

If the DC voltage has returned to the range controllable by the VCESs, the disconnected non-critical loads should be reconnected to improve service reliability. During gridconnected operation, disconnected non-critical loads can be reconnected without any problem because the designed power rating of the GTC is assumed to be sufficient to balance the power. During grid-connected operation, the DC voltages are maintained in Zone 2. Therefore, a disconnected non-critical load is reconnected to the DC microgrid if the DC voltage is maintained in Zone 2 for a specific time $\left(T_{N L D, \text { recon }}\right)$. 


\section{- Non-critical load reconnection rule 1:}

$$
V_{d c} \in \text { Zone } 2 \text { for } T_{N L D, \text { recon }}
$$

However, during islanded operation, inappropriate reconnection of a non-critical load will cause a disconnection of non-critical loads. For instance, if a non-critical load is reconnected when the upward power reserve of the DC microgrid is smaller than the power consumption of that load, the energy sources will be unable to supply power to all loads, and thus the DC voltage will decrease. Consequently, the reconnected non-critical load or another non-critical load will be disconnected.

In order to avoid this type of unexpected situation, reconnection rules of non-critical loads for islanded operation should be developed with respect to the upward power reserve of the DC microgrid. In other words, a disconnected non-critical load can be reconnected only if the upward power reserve of the DC microgrid is greater than the maximum power consumption of the disconnected load. Since the power consumption of a load varies continuously, the maximum power consumption is used as the criterion for reconnection.

Even though the exact values of the upward power reserve is not measurable at loads, the minimum power reserve can be estimated from the measured DC voltage, as described in Section 2. In Zone 4, the ESS controls the DC voltage thus the power output of the ESS is unknown. And the power outputs of the DGs and BG are their maximum power outputs because the reference voltages of them are higher than that of the ESS. Therefore, the minimum upward power reserve of Zone 4 is zero. When the DC microgrid operates in Zone 1 or Zone 3, there are two possible power outputs for the ESS, depending on the stored energy. If the stored energy has reached its maximum, the output power of the ESS is zero. Otherwise, the output power of the ESS is $-P_{\text {ESS,rating. Therefore, the }}$ minimum upward power reserve of Zone 1 and Zone 3 is the rated power of the ESS.
Based on the minimum power reserve for each zone, the following reconnection rule for a non-critical load is proposed. If the DC voltage is maintained in Zone 1 or Zone 3 for a specific time $\left(T_{N L D, \text { recon }}\right)$, a disconnected noncritical load whose maximum power consumption is lower than $P_{E S S \text {,rating }}$ can be reconnected in islanded operation.

\section{- Non-critical load reconnection rule 2:}

$$
V_{d c} \in\{\text { Zone } 1 \bigcup \text { Zone } 3\} \text { for } T_{N L D, \text { recon }}
$$

\section{Operational Modes}

The operation of a DC microgrid can be divided into grid-connected operation and islanded operation, according to the state of the GTC. Each operational state can be subdivided into several operation modes, depending on the power mismatch between generation and loads, and the states of the VCESs. This section describes the operational modes in detail. Transitions between operational modes and relevant events are also discussed.

\subsection{Modes of grid-connected operation}

Fig. 5 shows the detailed operational modes for gridconnected operation and the transitions between them. The notation is summarized on the right-hand side of the figure. Since the capacity of the GTC is larger than the maximum power output of the DGs, the DC microgrid does not operate in Zone 1. There are five different operational modes, divided into two parts. The first part is general gridconnected operation, represented by GM 1. The second part pertains to transitions from islanded operation to general grid-connected operation, and comprises GM 2, GM 3, GM 4, and GM 5. If the ESS is fully charged (i.e., $\left.E_{E S S}=E_{\max }\right)$, the DC microgrid initiates grid-connected operation in GM 2, where the ESS and BG operate in standby mode. Otherwise, the DC microgrid initiates grid-

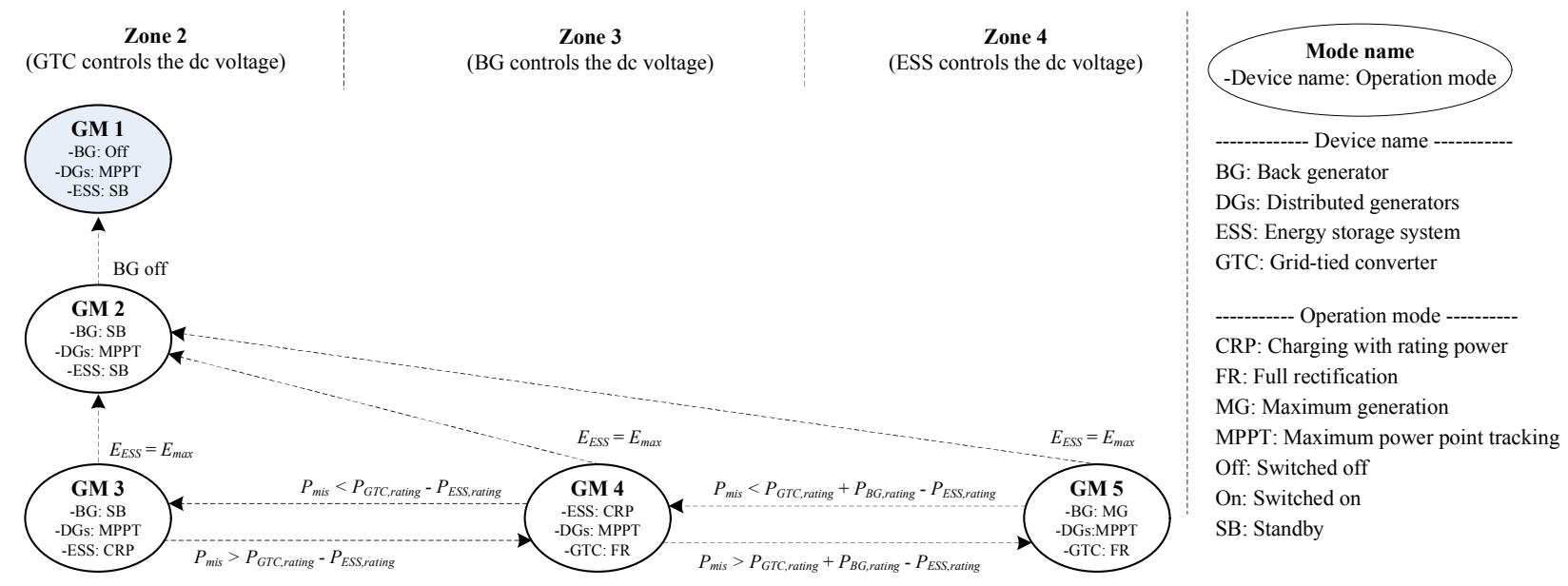

Fig. 5. Operational modes for grid-connected operation and transitions between them 
connected operation in GM 3, GM 4, or GM 5, depending on the power mismatch of the DC microgrid $\left(P_{m i s}\right)$, which is defined by

$$
P_{m i s}=P_{L D s}-P_{D G s, M P P T}
$$

where $P_{L D s}$ is the total power consumption of the loads and $P_{D G S, M P P T}$ is the total power output of the DGs when all DGs operate in the MPPT mode. The power charged to the ESS is its power rating in GM 3 or GM 4, or the maximum power that ensures an active power balance for the DC microgrid in GM 5. Once the energy stored in the ESS reaches its maximum limit, the operational mode of the DC microgrid is switched to GM 2. The DC microgrid operates in GM 2 until the "off" condition for the BG, described in (6), is satisfied. Finally, the BG is switched off and the operational mode of the DC microgrid is changed to the general grid-connected operational mode, GM 1.

\subsection{Modes of islanded operation}

Fig. 6 shows the detailed operational modes for islanded operation and the transitions between them. There are ten different operational modes in this case. In contrast to gridconnected operation, non-critical loads can be disconnected from the DC microgrid if the DC voltage is outside of the acceptable range, because the BG, ESS, and DGs lack balancing capability. If islanded operation begins with the BG switched off, the DC microgrid initially operates in IM 1, IM 2, IM 3, or IM 4 until the BG starts generating. After BG startup, the DC microgrid operates in IM 5, IM 6, IM 7, IM 8 , IM 9, or IM 10, depending on the power mismatch and the energy stored in the ESS. If the power mismatch increases, the operational mode changes from left to right.

\section{Parameter Determination Method}

In this section, we propose the methods to determine the control parameter settings, such as the range of operational zones, the voltage references for VCESs, and the disconnection voltages of the non-critical loads. The parameters are determined by solving the optimization problems (OPs) with the consideration of practical voltage drops.

\subsection{Operation constraints for each zone}

Firstly, constraints of the OPs for each operational zone are described. The common operation constraints applied to all operational zones are as follows

$$
\begin{gathered}
0 \leq P_{L D, i} \leq P_{L D, \max , i} \quad \text { for all load } i \\
P_{k}-V_{k} \sum_{i=1}^{n} G_{i k}\left(V_{k}-V_{i}\right)=0 \quad \text { for all bus } k
\end{gathered}
$$

where $P_{L D, i}$ is the power consumption of load $i, P_{k}$ is the net injected power at bus $k, V_{k}$ is the voltage of bus $k$, and $G_{i k}$ is a component of the conductance matrix. The second constraint, (12), corresponds to the power balance equation. From Figs. 5 and 6, the constraints for the power output and terminal voltage of the VCESs can be written as follows according to the operational zone.

- Zone 1:

$$
\begin{gathered}
P_{G T C}=P_{B G}=0 \\
P_{E S S}=0 \text { or }-P_{E S S, \text { rating }} \\
0 \leq P_{D G, j} \leq P_{D G, j, \text { rating }}
\end{gathered}
$$

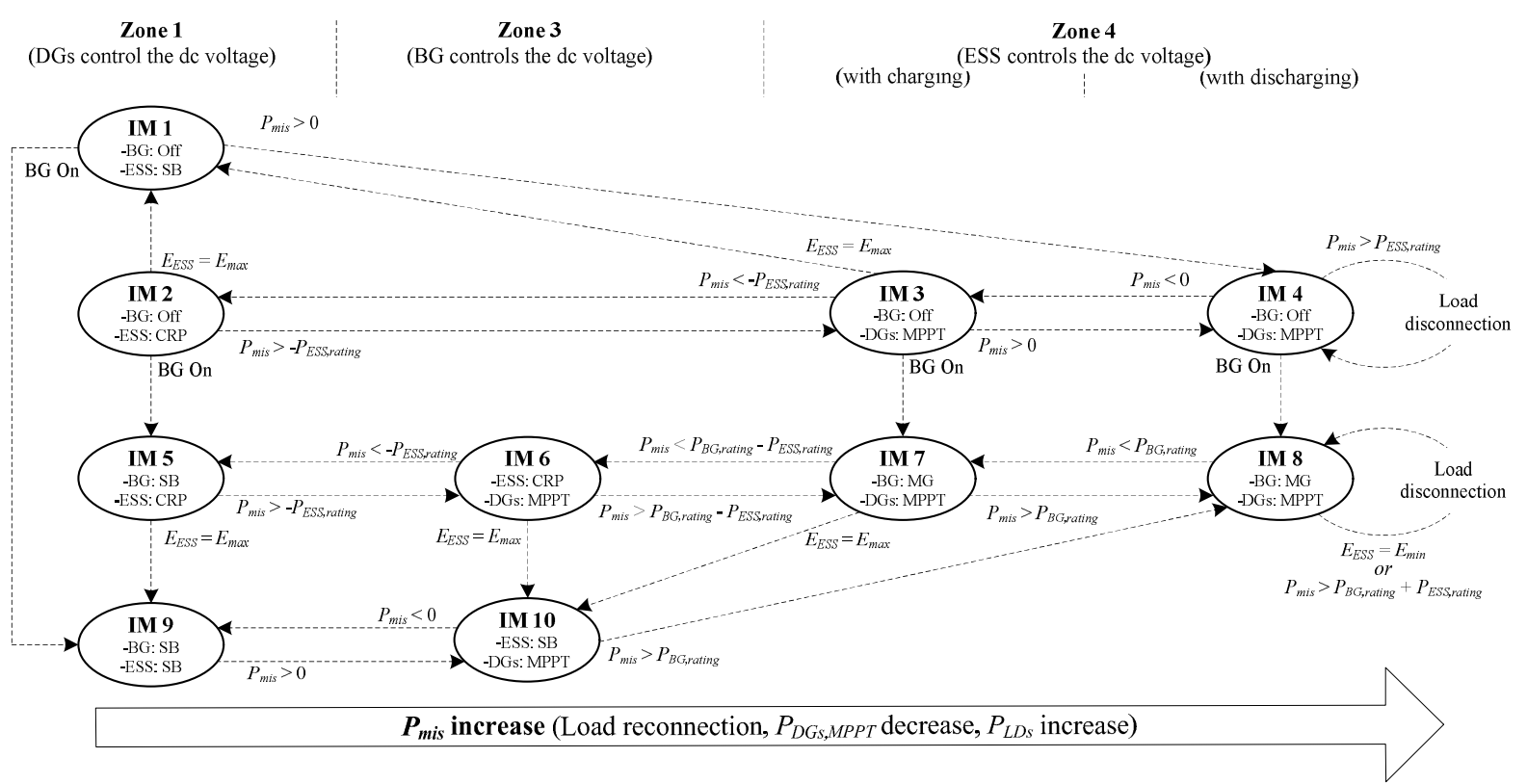

Fig. 6. Operational modes for islanded operation and transitions between them 


$$
\begin{gathered}
V_{k} \leq V_{D G s}^{*} \text { for } k \in S_{D G} \\
\prod_{k \in S_{D G}}\left(V_{k}-V_{D G s}^{*}\right)=0
\end{gathered}
$$

\section{- Zone 2:}

$$
\begin{aligned}
& -P_{G T C, \text { rating }} \leq P_{G T C} \leq P_{G T C, \text { rating }} \\
& P_{B G}=0 \\
& P_{E S S}=0 \text { or }-P_{E S S, \text { rating }} \\
& 0 \leq P_{D G, j} \leq P_{D G, j, \text { rating }} \\
& V_{k}=V_{G T C}^{*} \quad \text { for GTC connected bus } k
\end{aligned}
$$

- Zone 3:

$$
\begin{aligned}
& P_{G T C}=0 \\
& 0 \leq P_{B G} \leq P_{B G, \text { rating }} \\
& P_{E S S}=0 \text { or }-P_{E S S, \text { rating }} \\
& 0 \leq P_{D G, j} \leq P_{D G, j, \text { rating }} \\
& V_{k}=V_{B G}^{*} \quad \text { for BG connected bus } k
\end{aligned}
$$

\section{- Zone 4:}

$$
\begin{aligned}
& P_{G T C}=0 \\
& P_{B G}=0 \text { or } P_{B G, \text { rating }} \\
& -P_{E S S, \text { rating }} \leq P_{E S S} \leq P_{E S S, \text { rating }} \\
& 0 \leq P_{D G, j} \leq P_{D G, j, \text { rating }} \\
& V_{k}=V_{E S S}^{*} \quad \text { for ESS connected bus } k
\end{aligned}
$$

where $P_{G T C}, P_{B G}, P_{E S S}$, and $P_{D G, j}$ are the power outputs of the GTC, BG, ESS, and DG $j$, respectively, and $S_{D G}$ is a set of DG connected buses. In Zone 1, at least one of the terminal voltages of the DGs should be equal to the reference voltage and the others should be lower than or equal to the reference voltage. This constraint is represented by (16) and (17).

\subsection{Operation zones and voltage references for VCESs}

Firstly, the maximum and minimum operation voltage ranges of the DC microgrid are assumed to be defined as $V_{d c, \max }$ and $V_{d c, \min }$, respectively. Secondly, it is assumed that the DC voltage should be maintained as high as possible to reduce the loss of the system.

For Zone 1, the maximum voltage is simply set as $V_{d c, \text { max }}$ :

$$
V_{\text {Zone } 1, \max }=V_{d c, \max } .
$$

Since the DGs are the only VCES that supplies the power into the grid in Zone 1, the reference voltage of the DGs can be set as the same as the maximum voltage of
Zone 1. Considering the error in voltage measurement, the voltage reference for all DGs is determined as

$$
V_{D G s}^{*}=V_{\text {Zonel, } \max }-V_{\text {error, } \max } .
$$

where $V_{\text {error,max }}$ is the maximum voltage measurement error.

With given $V^{*} D G s$, the minimum voltage of the DC microgrid depends on various system conditions, such as the power output of energy sources and power demand of loads. The minimum voltage can be found by solving the OP given as follows:

$$
\underset{[P]}{\operatorname{Minimize}} \quad f=\min _{[k]} V_{k}
$$

where $P$ is the set of decision vector of the OP that describe various system conditions, and the constraints are (11)-(17).

With the optimum value of the OP, $f_{\text {opti }}$, the minimum voltage of Zone $1\left(V_{\text {Zonel,min }}\right)$, which is equal to the maximum voltage of Zone $2\left(V_{\text {Zone2,max }}\right)$, is determined as

$$
V_{\text {Zone1,min }}=V_{\text {Zone } 2, \max }=f_{\text {opti }}-V_{\text {error }, \max } .
$$

Unlike $V_{D G s}^{*}, V_{G T C}^{*}$ cannot be directly calculated from $V_{\text {Zone2,max }}$, since both the GTC and DGs can supply power into the DC micro-grid in Zone 2. In the proposed method, $V_{G T C}^{*}$ is calculated by solving an OP iteratively. The objective function of the OP is to maximize the voltage of the DC microgrid for a given $V_{G T C}^{*}$ :

$$
\underset{[P]}{\operatorname{Maximize}} f=\max _{[k]} V_{k} .
$$

In this OP, Eqs. (11), (12), and (18) - (22) are used as constraints. Initially, $V_{G T C}^{*}$ is set at

$$
V_{G T C}^{*}=V_{\text {Zone } 2, \max }-\Delta V
$$

where $\Delta V$ is an positive constant. Since the voltage measurement error is not considered in the calculated maximum voltage, $f_{\text {opti }}$ is compared to $V_{\text {Zone2,max }}-V_{\text {error,max }}$. If $f_{\text {opti }}$ is larger than $V_{\text {Zone2,max }}-V_{\text {error,max }}$, the voltage of the DC microgrid can be measured larger than $V_{\text {Zone2,max }}$ with given $V_{G T C}^{*}$. Therefore, $V_{G T C}^{*}$ is reduced by $\Delta V$ and the OP should be solved again. The voltage ranges of Zones 3 and 4 , and the reference voltages of $B G$ and ESS can be determined by similar procedure, as shown in Fig. 7.

\subsection{Disconnection voltage of the non-critical load}

Fig. 8 summarizes the proposed method to determine the disconnection voltages for the non-critical loads. It is assumed that the list of the order of disconnection among the non-critical loads or group of loads is given. For the first group of the loads in the list, the disconnection voltage can be determined by (39) considering the voltage measurement error. 


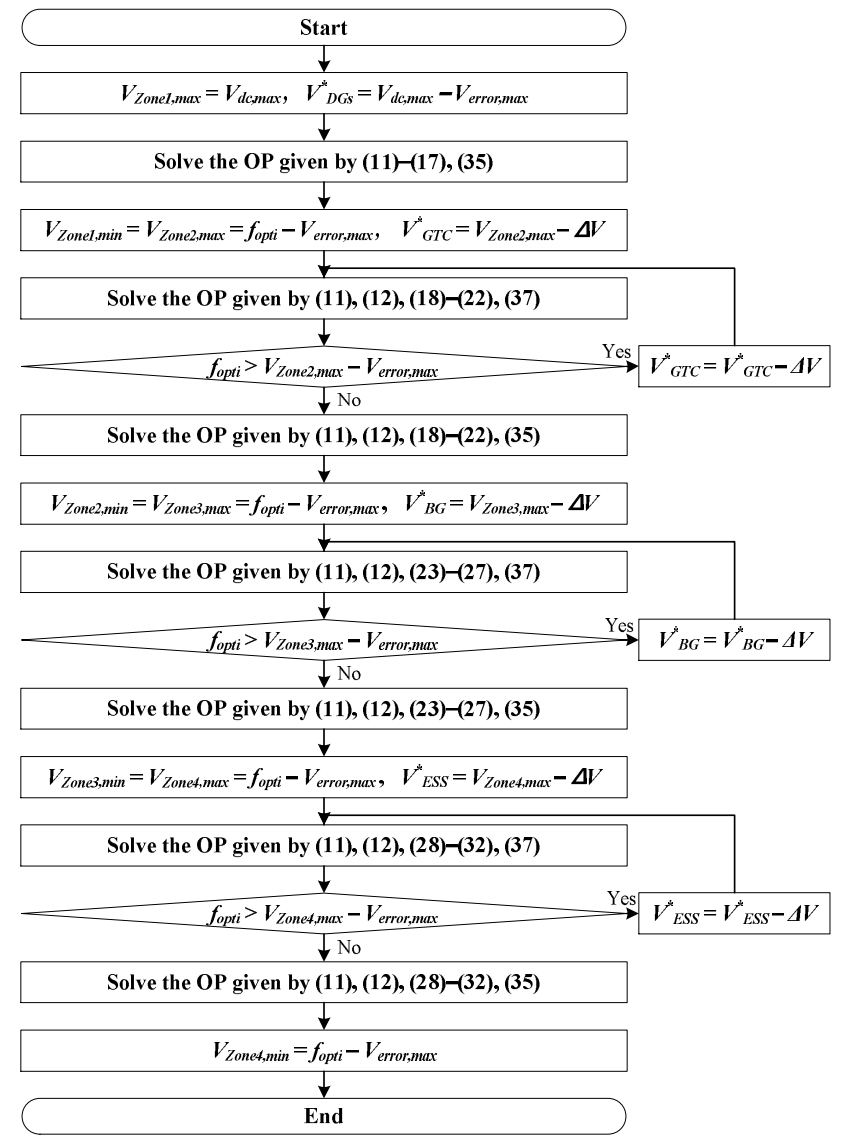

Fig. 7. Determination method for ranges of operation zones and voltage references for VCESs

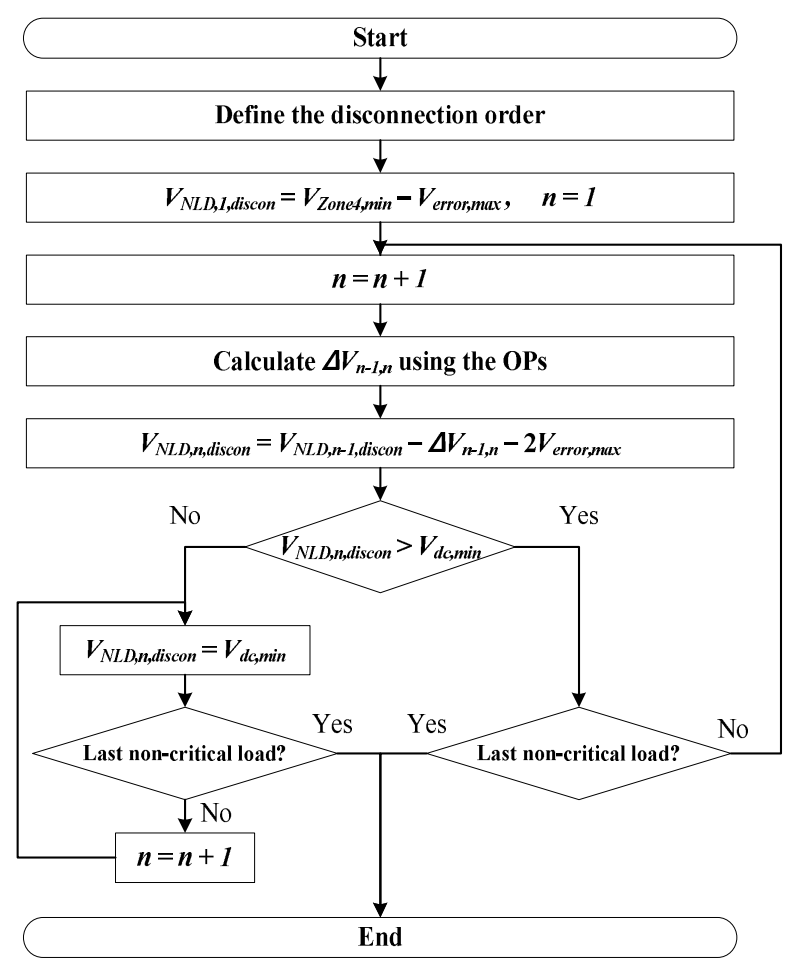

Fig. 8. Disconnection voltage calculation for the noncritical loads

$$
V_{N L D, 1, \text { discon }}=V_{\text {Zone4, } \min }-V_{\text {error }, \max }
$$

The disconnection voltages of the other non-critical loads or load groups should be set carefully, to guarantee that the non-critical loads are disconnected in a predetermined sequence. The difference in the disconnection voltage settings of two consecutive loads in the list should be larger than the maximum voltage difference between the buses where the loads are connected. The maximum voltage difference $\left(\Delta V_{n-1, n}\right)$ between the buses where the $(n-1)$ 'th and n'th order loads are connected can be calculated by solving the OP given by

$$
\underset{[P]}{\operatorname{Maximize}} \quad f=V_{N L D, n-1}-V_{N L D, n}
$$

where $V_{N L D, n-1}$ and $V_{N L D, n}$ are the voltages of the buses where the (n-1)'th and n'th order loads are connected. Using (11) - (32) and (40), maximum voltage differences for each zone can be calculated. Among them, the largest values will be used as the maximum voltage difference between the buses. If the calculated disconnection voltage setting is lower than the minimum operation voltage of the DC microgrid, $V_{d c, \text { min }}$, the disconnection voltage of the loads should be set as $V_{d c \text {,min }}$ to prevent a severe undervoltage violation.

\section{Simulations and Results}

In order to demonstrate the performance and validate the effectiveness of the proposed operational method, we have simulated various scenarios using the PSCAD/EMTDC program. In this paper, two simulation results that best illustrate the effectiveness of the proposed operational method are presented.

\subsection{Test system and scenarios}

Fig. 9 shows a single-line diagram of the $400-\mathrm{V}$ DC microgrid test system, which is connected to an AC system via a 55-kW GTC. The DC microgrid consists of an ESS, a BG, two DGs, one critical load, and two non-critical loads. The power rating of each component and the line resistance are shown in the figure. In order to easily demonstrate the effect of the energy stored in the ESS within the limited simulation time, we assume a small ESS capacity and a short BG startup time. The energy capacity of the ESS is $35 \mathrm{~kJ}$, and $E_{\min }$ and $E_{\max }$ are set at $10 \%$ and $95 \%$, respectively. Therefore, the ESS can supply power to the critical load for 1.5 seconds. The startup time for the backup generator is assumed to be 0.5 second.

It is assumed that the DC microgrid voltage is maintained in $\pm 5 \%$ range (i.e., $V_{d c, \max }=420 \mathrm{~V}$ and $V_{d c, \min }=380 \mathrm{~V}$ ). The voltage ranges of the operational zones and the reference voltages of the VCESs are determined based on the 


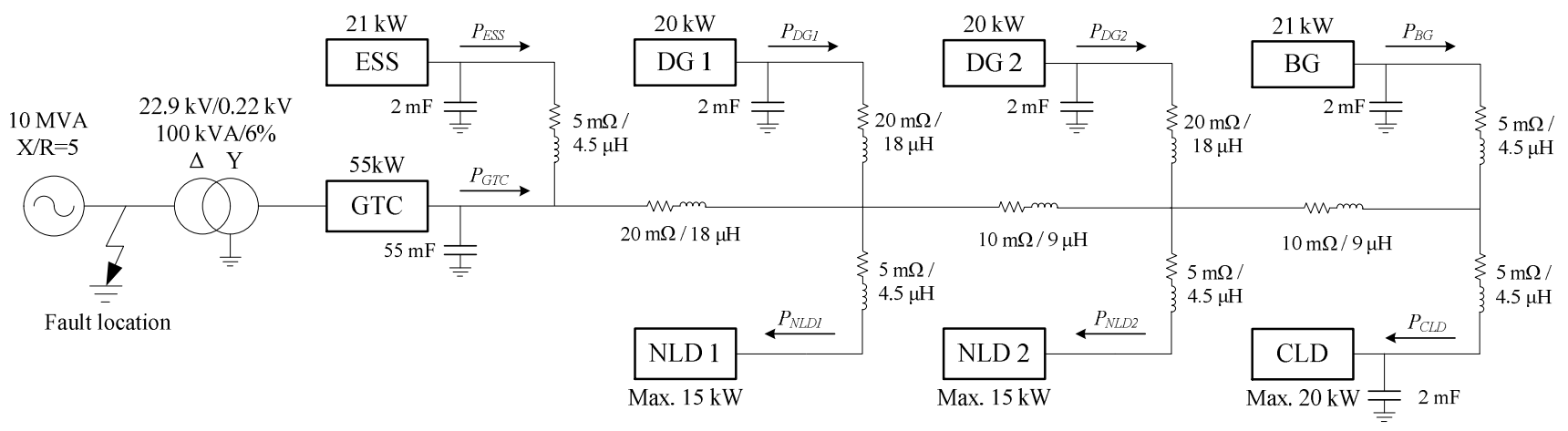

Fig. 9. DC microgrid test system (CLD: critical load, NLD: non-critical load)

Table 1. Voltage ranges of the operational zones and voltage references for the corresponding VCESs

\begin{tabular}{|c|c|c|c|}
\hline \multirow{2}{*}{ Zone } & \multirow{2}{*}{$\begin{array}{l}\text { Voltage reference } \\
\text { (V) }\end{array}$} & \multicolumn{2}{|c|}{ Voltage range $(\mathrm{V})$} \\
\hline & & Maximum & Minimum \\
\hline 1 & $V_{D G s}^{*}=419.0$ & 420.0 & 415.3 \\
\hline 2 & $V_{G T C}^{*}=410.3$ & 415.3 & 405.3 \\
\hline 3 & $V_{B G}^{*}=402.3$ & 405.3 & 398.3 \\
\hline 4 & $V_{E S S}^{*}=393.3$ & 398.3 & 389.3 \\
\hline
\end{tabular}

Table 2. Parameters for state control

\begin{tabular}{|c|c|c|c|}
\hline \multicolumn{4}{|c|}{ Backup generator } \\
\hline \multicolumn{2}{|c|}{$T_{B G \text {,on }}$} & \multicolumn{2}{|c|}{$T_{B G, \text { off }}$} \\
\hline \multicolumn{2}{|c|}{$0.6 \mathrm{~s}$} & \multicolumn{2}{|c|}{$1.5 \mathrm{~s}$} \\
\hline \multicolumn{4}{|c|}{ Non-critical load } \\
\hline & $V_{L D, \text { discon }}$ & $T_{N L D, \text { discon }}$ & $T_{N L D, \text { recon }}$ \\
\hline NLD 1 & $388.3 \mathrm{~V}$ & $0.01 \mathrm{~s}$ & $0.2 \mathrm{~s}$ \\
\hline NLD 2 & $385.2 \mathrm{~V}$ & $0.01 \mathrm{~s}$ & $0.1 \mathrm{~s}$ \\
\hline
\end{tabular}

Table 3. Initial states for each case

\begin{tabular}{c|c|c}
\hline & Case 1 & Case 2 \\
\hline$P_{G T C}$ & $28 \mathrm{~kW}$ & Off \\
\hline$E_{E S S}$ & $95 \%$ & $95 \%$ \\
\hline$P_{B G}$ & Off & $10 \mathrm{~kW}$ \\
\hline$P_{D G 1}$ & $9 \mathrm{~kW}$ & $18 \mathrm{~kW}$ \\
\hline$P_{D G 2}$ & $6 \mathrm{~kW}$ & $10 \mathrm{~kW}$ \\
\hline$P_{C L D}$ & $18 \mathrm{~kW}$ & $18 \mathrm{~kW}$ \\
\hline$P_{N L D 1}$ & $14 \mathrm{~kW}$ & $14 \mathrm{~kW}$ \\
\hline$P_{N L D 2}$ & $11 \mathrm{~kW}$ & $6 \mathrm{~kW}$ \\
\hline
\end{tabular}

proposed method and summarized in Table 1. The state control parameters for the BG and non-critical loads are listed in Table 2. For $V_{\text {errormax }}$ and $\Delta V, 1 \mathrm{~V}$ was used. The optimum values of the OPs were found by using a genetic algorithm [21].

The initial states of the components for the two simulation cases are summarized in Table 3. Case 1 demonstrates the islanding sequence of the microgrid, and how power is supplied to the non-critical loads during islanded operation. Case 2 demonstrates the operation mode change during islanded operation and the transition from islanded operation to grid-connected operation.

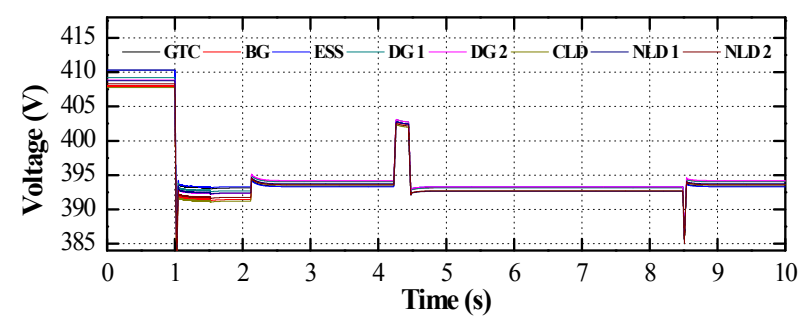

(a) DC voltages of the microgrid

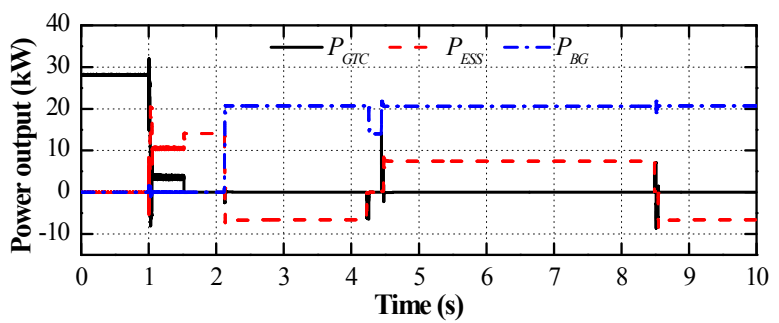

(b) Power outputs of the grid-tied converter, energy storage system, and backup generator

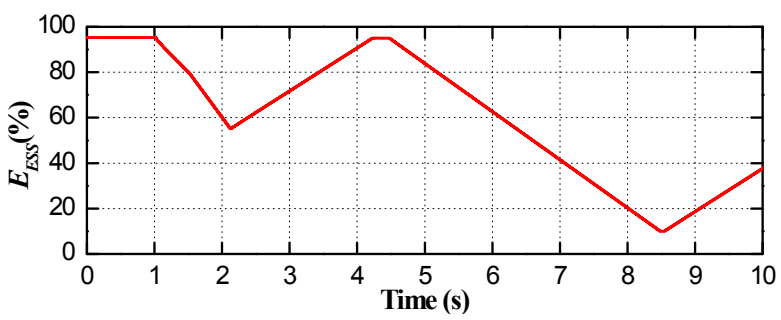

(c) Energy stored in the ESS

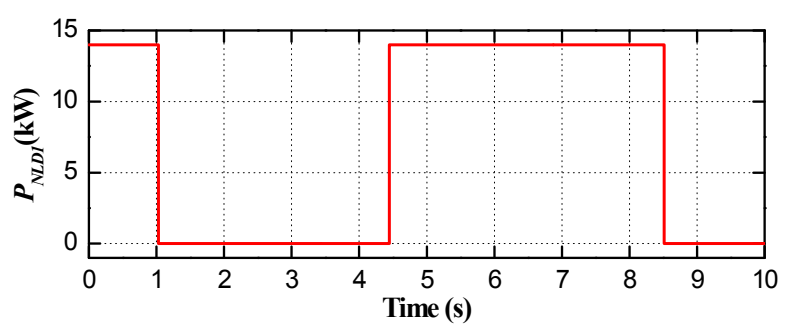

(d) Power consumption of non-critical load 1

Fig. 10. Simulation results for Case 1 


\subsection{Results for case 1}

Fig. 10 shows the simulation results for Case 1. Initially, the microgrid was connected to the main AC grid and operated in GM 1 mode. The DC voltages were maintained in Zone 2 by the GTC. In this simulation, it was assumed that a three-phase fault in the AC grid (see Fig. 9) was initiated at $1.0 \mathrm{~s}$, and GTC was switched off (the DC microgrid started islanded operation) at $1.5 \mathrm{~s}$.

At $1.0 \mathrm{~s}$, the DC voltage started to decrease since the grid fault prevented the GTC from controlling the voltage. After a while, the DC voltage decreased to $V_{E S S}^{*}$, and the ESS started supplying power to control the DC voltage. However, even when the ESS power output was at its maximum, the loads could not be fully satisfied by the ESS and DGs. Therefore, the DC voltage continued to decrease. After a while, the non-critical load 1 was disconnected because the DC voltage was lower than $388.3 \mathrm{~V}$ for $0.01 \mathrm{~s}$. After disconnection of this load, the DC voltage was controlled by the ESS and maintained in Zone 4. At $1.5 \mathrm{~s}$, the GTC was switched off and the microgrid was operated in IM 4 mode.

At $2.1 \mathrm{~s}$, i.e. $1.1 \mathrm{~s}\left(T_{B G \text {,on }}+T_{B G \text {,start }}=1.1\right)$ after the fault, the BG started to supply power. Between $2.1 \mathrm{~s}$ and $4.2 \mathrm{~s}$, the BG supplied its maximum power, and the remaining power was used to charge the ESS. During this period, the DC voltage was still controlled by the ESS in Zone 4, and the microgrid operational mode was IM 7. At $4.2 \mathrm{~s}$, when the ESS was fully charged, the operational mode of the microgrid changed to IM 10. The DC voltage was controlled by the BG in Zone 3.

At $4.5 \mathrm{~s}$, it was possible to reconnect non-critical load 1 since load reconnection rule 2 was satisfied (i.e., the DC voltage was in Zone 3 for $0.2 \mathrm{~s}$ ). After load reconnection, the ESS started to discharge since the loads could not be fully satisfied by the BG and DGs. Therefore, the DC voltages were in Zone 4, and the operational mode was IM 8. This operational status continued until the energy stored in the ESS reached its minimum limit and thus the ESS stopped discharging at $8.5 \mathrm{~s}$. At that time, non-critical load 1 was again disconnected since the BG and DGs could not supply power to all the loads. This same pattern of disconnection and reconnection would be repeated until other conditions changed. To summarize, the simulation results for Case 1 demonstrate that the microgrid can be smoothly islanded via the proposed operational strategies. Moreover, even though some non-critical loads must be shed to ensure stable operation, they can be reconnected to the microgrid, and thus service reliability is improved.

\subsection{Results for case 2}

Fig. 11 shows the simulation results for Case 2. Initially, the microgrid was islanded and operated in IM 10 mode (i.e. the ESS was in standby state, the DGs operated in the MPPT mode, and the DC voltage was controlled by the BG

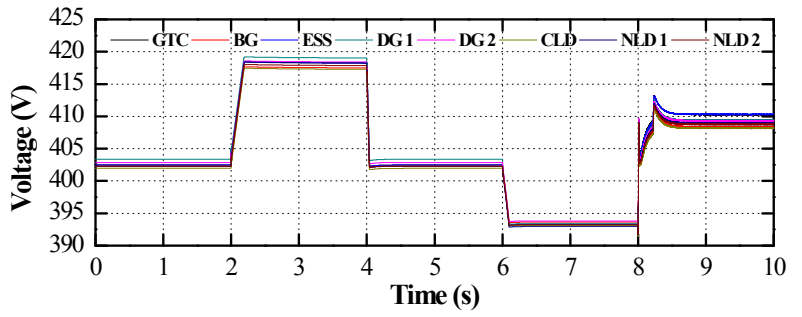

(a) DC voltages of the microgrid

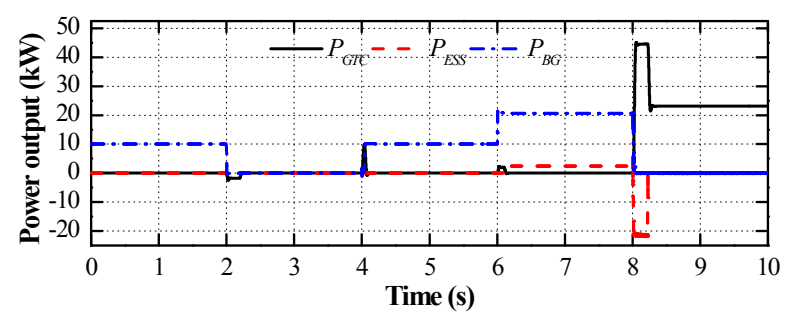

(b) Power outputs of the grid-tied converter, energy storage system, and backup generator

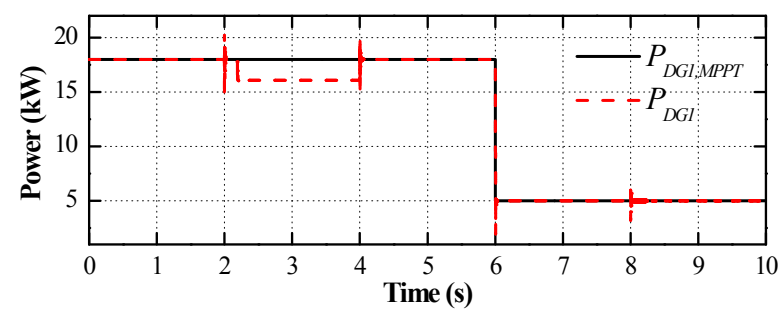

(c) Power output of distributed generator 1

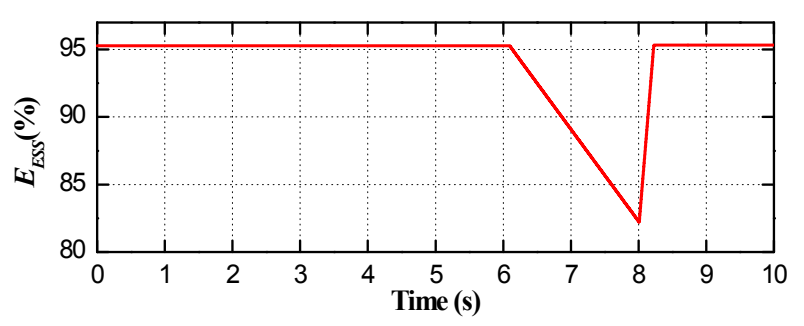

(d) Energy stored in the ESS

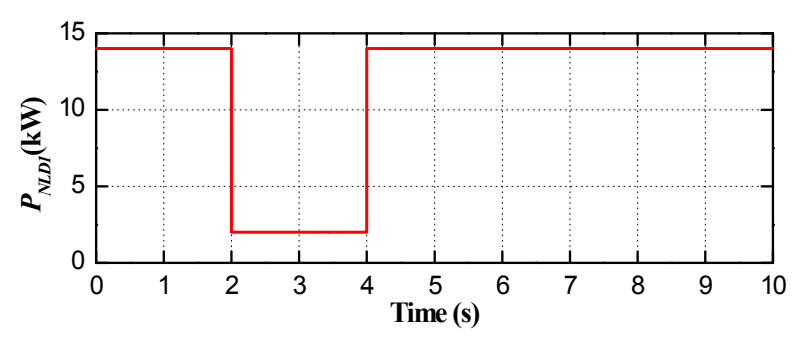

(e) Power consumption of non-critical load 1

Fig. 11. Simulation results for Case 2

in Zone 3). The simulation scenario is as follows.

- $2.0 \mathrm{~s}$ : The power consumption of non-critical load 1 $\left(P_{N L D I}\right)$ is decreased from 14.0 to $2.0 \mathrm{~kW}$.

- $4.0 \mathrm{~s}: P_{N L D I}$ is increased to $14.0 \mathrm{~kW}$.

- $6.0 \mathrm{~s}$ : The maximum power output of the DG 1, 
$P_{D G I, M P P T}$, decreased from 18.0 to $5.0 \mathrm{~kW}$.

- $8.0 \mathrm{~s}$ : The operation mode of the DC microgrid changed from islanded operation to grid-connected operation.

When $P_{N L D I}$ was decreased at $2.0 \mathrm{~s}$, the sum of the loads became smaller than the power output of the DGs, even though the BG supplied no power. As a result, the DC voltages of the DG1-connected bus started to increase and reached $419 \mathrm{~V}$, reference voltage, at $2.2 \mathrm{~s}$. Therefore, the DG 1 controlled the DC voltage by reducing its power output as shown in Fig. 11 (c) and the microgrid operated in IM 9 mode.

At $4.0 \mathrm{~s}$, the DGs could not fully supply the loads and the DC voltage started to decrease because $P_{N L D I}$ increased to $14.0 \mathrm{~kW}$. After a while, the $\mathrm{BG}$ controlled the $\mathrm{DC}$ voltage and the microgrid operated in IM 10 mode.

When $P_{D G 1, M P P T}$ decreased to $5 \mathrm{~kW}$ at $6.0 \mathrm{~s}$, the $\mathrm{BG}$ and DGs could not fully satisfy the loads, and thus the DC voltages started to decrease. After a while, the DC voltage decreased to the ESS reference voltage, and the ESS started to discharge. Therefore, the microgrid operated in IM 8 mode, and the DC voltage was controlled by the ESS in Zone 4.

At $8.0 \mathrm{~s}$, the microgrid was connected to the main grid. Since the GTC was able to supply the loads and the rated power of the ESS, the ESS charged with its rated power and the microgrid operated in GM 3 mode. After the energy stored in the ESS reached its maximum limit at 8.2 $\mathrm{s}$, the operational mode was changed to GM 2. Finally, the BG switched off at $9.7 \mathrm{~s}$ and thus the operational mode was changed to GM 1, the general grid-connected operation.

\section{Conclusions}

A decentralized control and operational strategy was proposed for a DC microgrid to ensure an uninterrupted power supply to critical loads, and to improve service reliability for non-critical loads. In order to implement the proposed operational strategy, DBS with a PI voltage controller was employed. The operational characteristics of DBS were first analyzed using a simplified DC microgrid system. From this analysis, we inferred that the minimum downward and upward power reserves of a DC microgrid can be determined for each operational zone. Based on the analyzed DBS characteristics, we specified the operational zones for the DC voltages, and proposed state control rules for the $\mathrm{BG}$, and disconnection rule for the non-critical loads to ensure an uninterrupted power supply to the critical loads. To improve service reliability of non-critical loads, the reconnection rule for the non-critical loads was also proposed. The detailed operational modes of a DC microgrid were described. The methods to determine the operational zones, voltage references for VCESs, state control parameters for the BG and non-critical load were proposed. Finally, the performance of the proposed operational strategy was verified by PSCAD/EMTDC simulations. The simulation results showed that service reliability for non-critical loads can be improved by using the proposed operational strategies.

\section{Acknowledgements}

This work was partially supported by Korea Institute of Energy Technology Evaluation and Planning (KETEP) grant funded by Korea Government Ministry of Trade, Industry and Energy (No. 20123010020080). This work was also partially supported by the National Research Foundation of Korea (NRF) grant funded by the Korea Government (MSIP) (No. 2010-0028509).

\section{References}

[1] H. Jiayi, J. Chuanwen, and X. Rong, "A review on distributed energy resources and microgrid," Renewable and Sustainable Energy Reviews, vol. 12, no. 9, pp. 2472-2483, Dec. 2008.

[2] J. M. Carrasco, L. G. Franquelo, J. T. Bialasiewicz, E. Galvan, R. C. P. Guisado, M. A. M. Prats, J. I. Leon, and N. Moreno-Alfonso, "Power-electronic systems for the grid integration of renewable energy sources: A survey," IEEE Trans. Ind. Electron, vol. 53, no. 4, pp. 1002-1016, June 2006.

[3] G. Pepermans, J. Driesen, D. Haeseldonckx, R. Belmans, and W. D'haeseleer, "Distributed generation: definition, benefits and issues," Energy Policy, vol. 33, no. 6, pp. 787-798, Jan. 2005.

[4] P. Piagi and R. H. Lasseter, "Microgrid: A conceptual solution," in Proceedings of the 2004 IEEE 35th Annual Power Electronics Specialists Conference, Aachen, Germany, June 2004.

[5] S. J. Ahn, J. W. Park, I. Y. Chung, S. I. Moon, S. H. Kang, and S. R. Nam, "Power-sharing method of multiple distributed generators considering control modes and configurations of a microgrid," IEEE Trans. Power Delivery, vol. 25, no. 3, pp. 2007-2016, July 2010.

[6] E. Serban and H. Serban, "A control strategy for distributed power generation microgrid application with voltage- and current-controlled source converter," IEEE Trans. Power Electron, vol. 25, no. 12, pp. 2981-2992, Dec. 2010.

[7] X. F. Wang, J. M. Guerrero, Z. Chen and F. Blaabjerg, "Distributed energy resources in grid interactive AC microgrids," in Proceedings of the 2010 2nd IEEE International Symposium on Power Electronics for Distributed Generation Systems, Hefei, China, June 2010.

[8] J. M. Guerrero, J. C. Vasquez, J. Matas, L. G. Vicuna, and M. Castilla, "Hierarchical control of droop-con- 
trolled AC and DC microgrids - A general approach toward standardization," IEEE Trans. Ind. Electron, vol. 58, no. 1, pp. 158-172, Jan. 2011.

[9] J. H. Lee, H. J. Kim, B. M. Han, Y. S. Jeong, H. S. Yang, and H. J. Cha, "DC micro-grid operational analysis with a detailed simulation model for distributed generation," Journal of Power Electronics, vol. 11, no. 3, pp. 350-359, May 2011.

[10] J. Schonberger, R. Duke, and S. D. Round, "DC-bus signaling: A distributed control strategy for a hybrid renewable nanogrid," IEEE Trans. Ind. Electron, vol. 53, no. 5, pp. 1453-1460, Oct. 2006.

[11] D. Salomonsson, L. Soder, and A. Sannino, "An adaptive control system for a dc microgrid for data centers," IEEE Trans. Ind. Applications, vol. 44, no. 6, pp. 1910-1917, Nov. 2008.

[12] H. Kakigano, Y. Miura, T. Ise, and R. Uchida, "DC micro-grid for super high quality distribution - System configuration and control of distributed generations and energy storage devices," in Proceedings of the 37th Annual IEEE Power Electronics Specialists Conference, Jeju, Korea, June 2006.

[13] H. Kakigano, Y. Miura, and T. Ise, "Low-voltage bipolar-type DC microgrid for super high quality distribution," IEEE Trans. Power Electronics, vol. 25, no. 12, pp. 3066-3075, Dec. 2010.

[14] Y. Ito, Y. Zhongqing, and H. Akagi, "DC microgrid based distribution power generation system," in Proceedings of the 4th International Power Electronics and Motion Control Conference, Xi'an, China, August 2004.

[15] L. Xu and D. Chen, "Control and operation of a DC microgrid with variable generation and energy storage," IEEE Trans. Power Delivery, vol. 26, no. 4, pp. 2513-2522, Oct. 2011.

[16] D. Chen and L. Xu, "Autonomous DC voltage control of a DC microgrid with multiple slack terminals," IEEE Trans. Power System, vol. 27, no. 4, pp. 18971905, Nov. 2012.

[17] W. Tang and R. H. Lasseter, "An lvdc industrial power supply system without central control unit," in Proceedings of the 31th Annual IEEE Power Electronics Specialists Conference, Galway, Ireland, June 2000.

[18] K. Sun, L. Zhang, Y. Xing and J. M. Guerrero, "A Distributed control strategy based on DC bus signaling for modular photovoltaic generation systems with battery energy storage," IEEE Trans. Power Electronics, vol. 26, no. 10, pp. 3032-3045, Oct. 2011.

[19] N. Eghtedarpour and E. Farjah, "Control strategy for distributed integration of photovoltaic and energy storage systems in DC micro-grids," Renewable Energy, vol. 45, pp. 96-110, Sept. 2012.

[20] A. Sannino, G. Postiglione, and M. Bollen, "Feasibility of a dc network for commercial facilities," IEEE Trans. Ind. Applications, vol. 39, no. 5, pp.
1499-1507, Sept. 2003.

[21] Kwang Y. Lee, Mohamed A. El-Sharkawi, Modern Heuristic Optimization Techniques, IEEE Press, 2008.

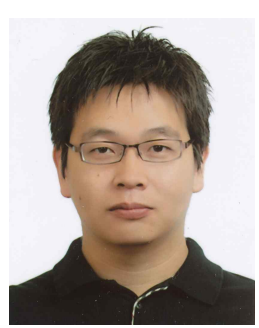

Pyeong-Ik Hwang He received the B.S. degree in Science and the integrated M.S. and Ph.D. degrees in Electrical Engineering from Seoul National University, Seoul, Korea in 2006 and 2014, respectively. Currently, he is a Research Professor at Korea University, Seoul, Korea. His research interests are distributed energy resources, distribution system operation, microgrids, and smart grids.

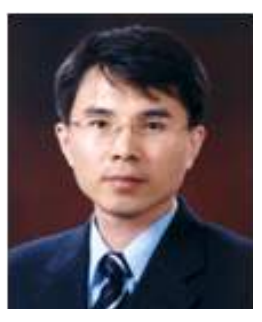

Gilsoo Jang He received the B.S. and M.S. degrees in Electrical Engineering from Korea University, Seoul, Korea, in 1991 and 1994, respectively, and the Ph.D. degree in Electrical and Computer Engineering from Iowa State University, Ames, in 1997. He was a Visiting Scientist at Iowa State University from 1997 to 1998 and a Senior Researcher with the Korea Electric Power Research Institute, Daejeon, Korea, from 1998 to 2000. Currently, he is a Professor in the School of Electrical Engineering, Korea University. His research interests include power quality and power system control.

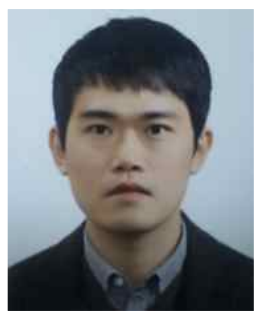

Gi-Chang Pyo He received the B.S., M.S., and Ph.D. degrees in Electrical Engineering from Seoul National University, Korea, in 2004, 2006, and 2013, respectively. Currently, he is a senior researcher in Samsung Heavy Industries Co., Ltd, Korea. His research interests are wind power generation, microgrids, smart grids, and real-time simulation.

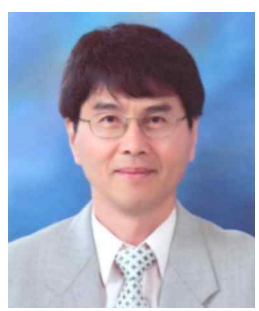

Byung-Moon Han He received the B.S. degree in Electrical Engineering from Seoul National University, Seoul, Korea, in 1976, and the M.S. and Ph.D. degrees from Arizona State University, USA, in 1988 and 1992, respectively. He was with the Westinghouse Electric Corporation as a Senior Research Engineer in the Science and Technology Center, Pittsburg, PA, USA. He is currently a Professor in the Department of Electrical Engineering, Myongji University, Seoul, Korea. 
His current research interests include power electronics applications for FACTS, custom power, distributed generation, and microgrid.

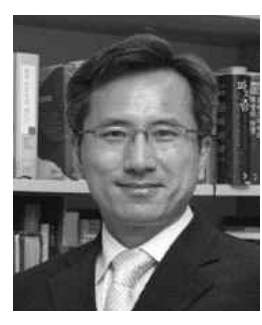

Seung-Il Moon He received the B.S. degree in Electrical Engineering from Seoul National University, Seoul, Korea, in 1985, and the M.S. and Ph.D. degrees in Electrical Engineering from Ohio State University, Columbus, $\mathrm{OH}$, USA in 1989 and 1993, respectively. He was an Assistant Professor of the Department of Electrical Engineering at Chonbuk National University. And currently he is a Professor of the School of Electrical Engineering and Computer Science at Seoul National University. $\mathrm{He}$ is a Member of Presidential Committee of Green Growth in Korea, Advisor for Ministry of Knowledge Economy in Korea, Member of Electric Regulatory Commission in Korea, Executive Director of Korea Institute of Electrical Engineers, and Executive Director of Korea Smart Grid Institute. His special fields of interest include smart grid, distributed generation, FACT system, renewable energy, and energy storage systems.

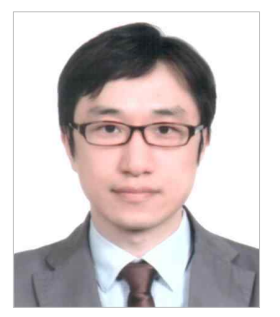

Seon-Ju Ahn He received the B.S., M.S., and Ph.D. degrees in Electrical Engineering from Seoul National University, Korea, in 2002, 2004, and 2009, respectively. Currently, he is an Assistant Professor at Chonnam National University, Gwangju, Korea. His research interests are power quality, distributed energy resources, microgrids, smart grids, and real-time simulation. 